\title{
Cross talk between hedgehog and epithelial-mesenchymal transition pathways in gastric pit cells and in diffuse-type gastric
}

\section{cancers}

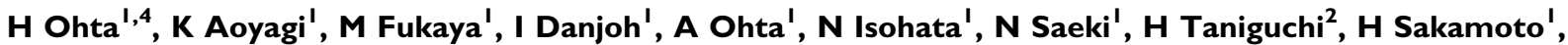 \\ T Shimoda ${ }^{3}$, T Tani $^{4}, \mathbf{T}$ Yoshida' and H Sasaki*, I
}

'Genetics Division, National Cancer Center Research Institute, I-I, Tsukiji 5-chome, Chuo-ku, Tokyo 104-0045, Japan; ${ }^{2}$ Pathology Division, National Cancer Center Research Institute, I-I, Tsukiji 5-chome, Chuo-ku, Tokyo 104-0045, Japan; ${ }^{3}$ Clinical Laboratory, National Cancer Center Hospital, I-I, Tsukiji 5-chome, Chuo-ku, Tokyo 104-0045, Japan; ${ }^{4}$ Department of Surgery, Shiga University of Medical Science, Seta Tsukinowa-cho, Otsu-shi, Shiga 520-2192, Japan

\begin{abstract}
We previously reported hedgehog $(\mathrm{Hh})$ signal activation in the mucus-secreting pit cell of the stomach and in diffuse-type gastric cancer (GC). Epithelial-mesenchymal transition (EMT) is known to be involved in tumour malignancy. However, little is known about whether and how both signallings cooperatively act in diffuse-type GC. By microarray and reverse transcription-PCR, we investigated the expression of those Hh and EMT signalling molecules in pit cells and in diffuse-type GCs. How both signallings act cooperatively in those cells was also investigated by the treatment of an Hh-signal inhibitor and siRNAs of Hh and EMT transcriptional key regulator genes on a mouse primary culture and on human GC cell lines. Pit cells and diffuse-type GCs co-expressed many Hh and EMT signalling genes. Mesenchymal-related genes (WNT5A, CDH2, PDGFRB, EDNRA, ROBOI, ROR2, and MEF2C) were found to be activated by an EMT regulator, SIPI/ZFHXIB/ZEB2, which was a target of a primary transcriptional regulator GLII in Hh signal. Furthermore, we identified two cancer-specific Hh targets, ELKI and MSX2, which have an essential role in GC cell growth. These findings suggest that the gastric pit cell exhibits mesenchymal-like gene expression, and that diffuse-type GC maintains expression through the Hh-EMT pathway. Our proposed extensive Hh-EMT signal pathway has the potential to an understanding of diffusetype GC and to the development of new drugs.
\end{abstract}

British Journal of Cancer (2009) I 00, 389-398. doi:10.1038/sj.bjc.6604846 www.bjcancer.com

Published online 23 December 2008

(c) 2009 Cancer Research UK

Keywords: gastric pit cell; diffuse-type gastric cancer; hedgehog; epithelial-mesenchymal transition; cancer-linked hypomethylation

Gastric cancer (GC) is one of the most frequent fatal malignancies in the world (Maxwell Parkin, 2001), and will be unresectable for more than two-thirds of its sufferers. Even patients with an operable tumour have a high rate of both local and distant recurrence with a 5-year survival rate of less than $30 \%$; furthermore, the response rate to chemotherapy in unresectable and recurrent cases is at most $40 \%$ (Wohrer et al, 2004; Lordick and Siewert, 2005). Gastric cancers are histologically classified into two major types: intestinal-type (clustered and well-differentiated, and glandular-like types) and diffuse-type (infiltrating, poorlydifferentiated, and scattered types) (Lauren, 1965; Tatematsu et al, 1990; Ming, 1998; Yuasa, 2003). Intestinal-type GC develops through some sequential stages including Helicobacter pylori (H. pylori)-associated gastritis, intestinal metaplasia (IM), and dysplasia. This type predominates in high-risk geographic areas, such as east Asia, showing a correlation with the prevalence in the region of $H$. pylori infection among the elderly. Diffuse-type GC, however, is more uniformly distributed geographically, is

*Correspondence: Dr H Sasaki; E-mail: hksasaki@ncc.go.jp

Received 26 August 2008; revised 25 November 2008; accepted 28 November 2008; published online 23 December 2008 apparently unrelated to $H$. pylori prevalence and typically develops from $H$. pylori-free, morphologically normal gastric mucosa without atrophic gastritis, or IM. A de novo diffuse-type GC is believed to develop from stem cells or precursors for gastric epithelial cells in the background of relatively normal gastric mucosa (Hohenberger and Gretschel, 2003; Schier and Wright, 2005). Unlike the decreasing incidence of the intestinal-type GC, the prevalence of the diffuse type is reportedly increasing worldwide (Crew and Neugut, 2006). Therefore, molecular characterisation of diffuse-type GC, especially on infiltrating and scattered type of growth or identification of cancer stem cell is important for the development of new drugs for this type of cancer. The infiltrating and scattered type of growth in diffuse-type GC has been reported to be mediated by loss of E-cadherin (CDH1) function through somatic mutation, promoter methylation, and cancer-associated downregulation (Kountouras et al, 2005). Recently, CDH1 has been reported to be downregulated by an epithelial-mesenchymal transition (EMT) regulator, TWIST, which plays an essential role in breast cancer metastasis, especially of the diffuse type (Yang et al, 2004). However, a more detailed signal network needs to be revealed to understand diffuse-type GC growth and progression. 
Recently, we reported that hedgehog (Hh) signal activation selectively occurs in diffuse-type GC and that the Hh signal block inhibits growth of GC cells with Hh activation (Fukaya et al, 2006). In mammals, Hh ligands are composed of three members: Sonic $\mathrm{Hh}(\mathrm{SHH})$, Indian $\mathrm{Hh}(\mathrm{IHH})$, and Desert $\mathrm{Hh}(\mathrm{DHH})$. Activation of Hh signalling is initiated through binding of any of the above three ligands to a 12-transmembrane protein receptor, $\mathrm{PTCH}$, which acts as a negative regulator of a 7-transmembrane protein, SMO. Binding between $\mathrm{Hh}$ and PTCH results in de-repression of SMO, thereby activating a cascade that leads to the translocation of the active form of the transcription factor GLI to the nucleus. Nuclear GLI activates expression of a variety of target genes such as BMP4, FOXA2, ISL1, and FOXM1 (Krishnan et al, 1997; Van den Brink et al, 2001; Teh et al, 2002). Recently, Ihog (interference Hh) and Boi, which de-repress SMO through interaction with Hh ligands, have been identified in Drosophila (Yao et al, 2006). Their mouse/ human homologues Boc/BOC and Cdo/CDO have the same functions (Tenzen et al, 2006; Yao et al, 2006; Zhang et al, 2006).

Here we report that an EMT regulator SIP1 is a target of the Hh signal in gastric pit cells and in diffuse-type GC, and that SIP1 regulates mesenchymal-related genes (WNT5A, CDH2, PDGFRB, EDNRA, ROBO1, ROR2, and MEF2C), which express preferentially in both pit cell and diffuse-type GC. Furthermore, we identified two cancer-specific Hh targets, ELK1 and MSX2, which have an essential role in tumour cell growth.

\section{MATERIALS AND METHODS}

\section{Tissue samples}

Gastric cancer and non-cancerous tissues were provided by the National Cancer Center Hospital after obtaining informed consent from each patient and approval by the Center's Ethics Committee. All cancer specimens were reviewed and classified histopathologically according to the Japanese Classification of Gastric Cancer. Tissue specimens were immediately frozen with liquid nitrogen after surgical extraction, and stored at $-80^{\circ} \mathrm{C}$ until use.

\section{Microarray analysis}

Total RNA was isolated by suspending the cells in an ISOGEN lysis buffer (Nippon Gene, Toyama, Japan), followed by precipitation with isopropanol. We used Human Expression Array U95A version 2 (Affymetrix, Santa Clara, CA, USA) for analysis of mRNA expression levels corresponding to 12600 transcripts. The procedures were conducted according to the supplier's protocols. The expression value (average difference; $A D$ ) of each gene was calculated using GeneChip Analysis Suite version 4.0 software (Affymetrix). The mean of $\mathrm{AD}$ values in each experiment was 1000 to reliably compare variable multiple arrays.

\section{Laser microdissection (LMD), RNA extraction, RT - PCR, and quantitative real-time PCR}

The cryostat sections $(8 \mu \mathrm{m})$ of frozen tissues were microdissected with a Pixcell II LCM system (Arcturus Engineering, Mountain View, CA, USA). Total RNA was isolated by suspending the cells in an ISOGEN lysis buffer (Nippon Gene), followed by precipitation with isopropanol. The mRNA was amplified by an efficient method of high-fidelity mRNA amplification, called TALPAT (T7 RNA polymerase promoter-attached, adaptor ligation-mediated, and PCR amplification followed by in vitro T7-transcription) (Aoyagi et al, 2003; Kobayashi et al, 2004; Nakamura et al, 2006). As described in our previous report (Fukaya et al, 2006), semiquantitative reverse transcription (RT) - PCR and quantitative real-time PCR were carried out using primer sets (Supplementary
Table 1). For semi-quantitative RT-PCR, we showed data within linear range by performing 25-35 cycles of PCR. For quantitative real-time PCR, the expression level of each mRNA was normalised with that of GAPDH mRNA.

\section{Immunohistochemistry}

Specimens fixed in formalin and embedded in paraffin were cut into $4 \mu \mathrm{m}$ sections, subsequently dewaxed, and dehydrated. Endogenous peroxidase activity was blocked with $3 \% \mathrm{H}_{2} \mathrm{O}_{2}$ in methanol for $30 \mathrm{~min}$ and endogenous biotin with a blocking kit (Vector Laboratories, Burlingame, CA, USA). Antigen retrieval was performed by autoclave for $10 \mathrm{~min}$ at $121^{\circ} \mathrm{C}$ in $10 \mathrm{~mm}$ citrate buffer, pH 6.0. Sections were blocked for DAKO protein block (DAKO, Carpinteria, CA, USA), and incubated overnight at $4{ }^{\circ} \mathrm{C}$ with diluted rabbit polyclonal antibody directed against human GLI1 (sc-20687, 1 : 100; Santa Cruz Biochemistry, Santa Cruz, CA, USA), GLI2 (ab7181, 1:200; Abcam Ltd, Cambridge, MA, USA), PDGFRB (sc-339, 1:200; Santa Cruz Biochemistry), EDNRA (E3651, 1:100; SIGMA, St Louis, MO, USA), and mouse monoclonal antibody directed against SMTN (MAB3242, 1:200; Chemicon International, Tamecula, CA, USA). The next day, after washing the sections with PBS containing $0.1 \%$ Tween-20, biotinylated secondary antibodies were added for $30 \mathrm{~min}$ at room temperature. Detection was carried out with the Vectastain ABC Elite Kit (Vector Laboratories). After extensive rinsing and incubation with an avidin-biotin immunoperoxidase complex, staining was visualised with the DAB system (Nichirei, Tokyo, Japan), and the sections were counter-stained with Mayer's hematoxylin.

\section{Cell lines and siRNA transfection}

Seven diffuse-type GC-derived cell lines, HSC39, HSC43, HSC44, HSC58, HSC59, HSC60, and KATOIII, and four intestinal-type GC-derived cell lines, MKN7, MKN28, MKN74, and HSC57 were maintained in RPMI1640 or Dulbecco's modified Eagle's medium supplemented with $10 \%$ fetal calf serum, $0.15 \%$ sodium bicarbonate, $2 \mathrm{~mm}$ L-glutamine, and penicillin-streptomycin. An $\mathrm{Hh}$ pathway-specific antagonist cyclopamine or tomatidine, an inactive but structurally related compound (Toronto Research Chemicals, North York, Ontario, Canada) dissolved in $100 \%$ ethanol was added to HSC60 at $30 \mu \mathrm{M}$. After 6, 12, and $24 \mathrm{~h}$, cells were collected and total RNA was isolated, respectively. Five siRNA fragments were designed for suppressing GLI1, GLI2, SIP1, $E L K 1$, and $M S X 2$ expression respectively, and the most effective one was selected by quantitative real-time RT - PCR analysis. The five siRNAs were GLI1 siRNA (SI00074802, QIAGEN, Valencia, CA, USA), GLI2 siRNA (SI02634842, QIAGEN), SIP1 siRNA (108633, Ambion, Austin, TX, USA), ELK1 siRNA (SI00300146, QIAGEN), and MSX2 siRNA (SI00038031, QIAGEN). These siRNAs were introduced to HSC60 using Dharma FECT1 ${ }^{\mathrm{TM}}$ (Dharmacon, La Fayette, CO, USA), following the procedure recommended by the manufacturer. The RT-PCR analysis was carried out at $24 \mathrm{~h}$ after siRNA transfection.

\section{Primary culture of mouse gastric epithelial cells}

On postnatal day 4 , the stomach of $57 \mathrm{BL} / 6 \mathrm{~J}$ mice was minced, then suspended and sterilised with $10 \%$ antibiotic-antimycotic drugs (Invitrogen, Carlsbad, CA, USA), and digested by incubation for $12 \mathrm{~min}$ at $37^{\circ} \mathrm{C}$ in $0.05 \%$. collagenase 1 (Nitta Gelatine, Osaka, Japan), and then manually dissociated with scissors, and incubated for $5 \mathrm{~min}$ at $37^{\circ} \mathrm{C}$ with a $1: 1$ ratio of $0.5 \%$ Trypsin-EDTA (Invitrogen) and Dispase 10000PU (Godo Shusei, Tokyo, Japan) in PBS (-). After being filtered with a $100 \mu \mathrm{m}$ cell strainer (Falcon, Franklin Lakes, NJ, USA), the digested tissue fragments were centrifuged for $5 \mathrm{~min}$ at 1200 r.p.m., resuspended in Defined Keratinocyte-SFM (Invitrogen) with $1 \mathrm{ml}$ of supplement and 


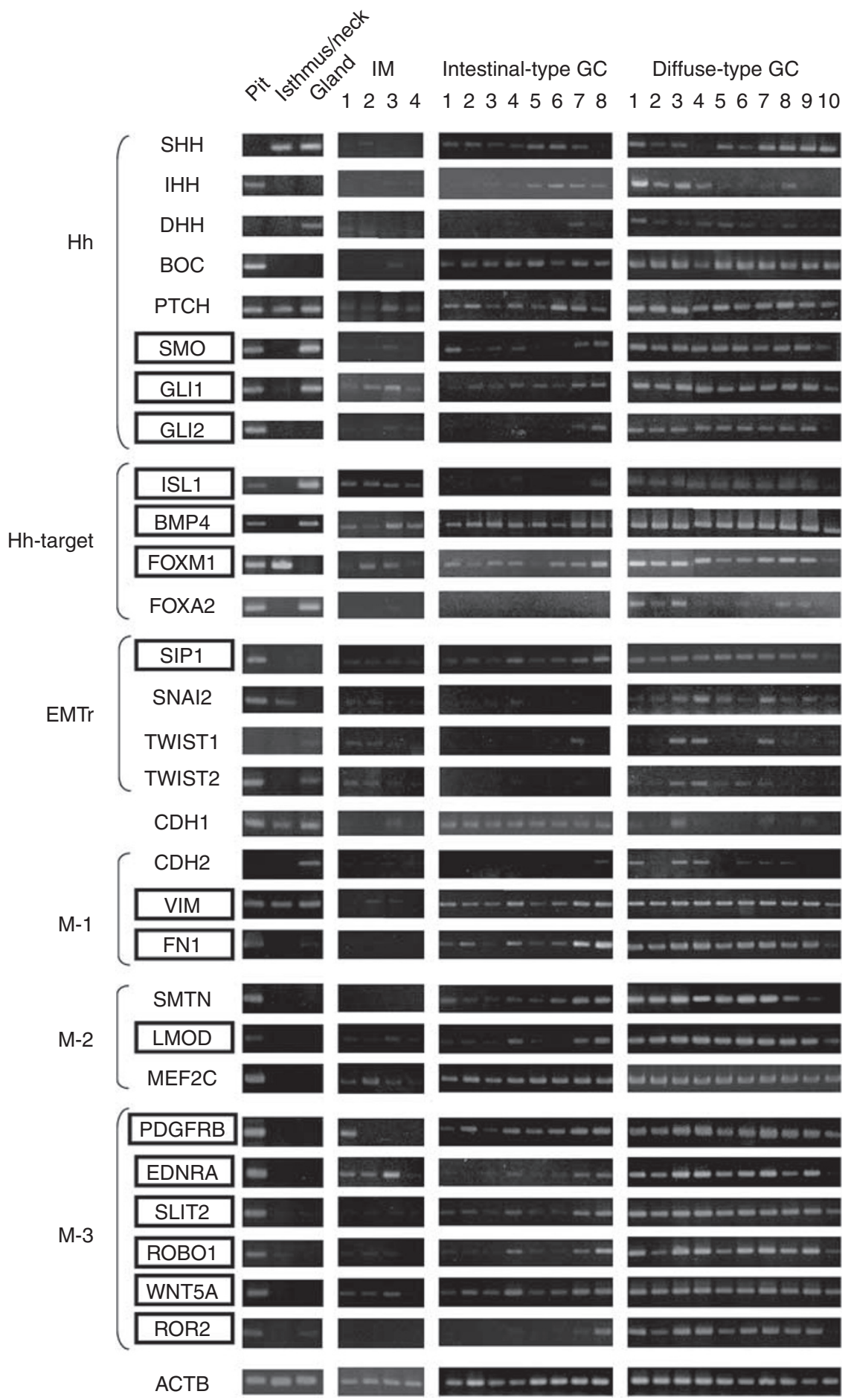

Figure I Semi-quantitative RT-PCR analyses of Hh signalling molecules, EMT regulators, and mesenchymal-related genes in three regions (pit, isthmus/ neck, and gland) of normal gastric mucosa, intestinal metaplasias (IMs), and two types of gastric cancers. Hh signalling molecules are expressed preferentially in diffuse-type gastric cancers compared with IMs and intestinal-type gastric cancers, as reported previously (Fukaya et al, 2006). Three EMT regulators (SIPI, SNAI2, and TWIST2) and IO mesenchymal-related genes (FNI, SMTN, LMOD, MEF2C, PDGFRB, EDNRA, SLIT2, ROBOI, WNT5A, and ROR2) are preferentially expressed in the pit region. These EMT regulators and mesenchymal-related genes are expressed highly and preferentially in diffuse-type gastric cancers compared with the IMs and intestinal-type gastric cancers. EMTr, EMT regulator; M-I, three typical mesenchymal genes; M-2, three muscle-related mesenchymal genes, and M-3, six other mesenchymal-related genes. GC, gastric cancer.

antibiotic-antimycotic, plated in type 1 collagen-coated $35 \mathrm{~mm}$ culture dishes (IWAKI, Tokyo, Japan) and incubated at $37^{\circ} \mathrm{C}$ in a humidified atmosphere flushed with $5 \% \mathrm{CO}_{2}$ in the air.
Twenty-four hours after plating, non-adhesive cells were discarded. At 1 day after culture, $10 \mu \mathrm{M}$ of cyclopamine or tomatidine was added. 


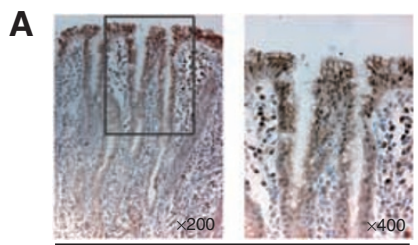

GLI1

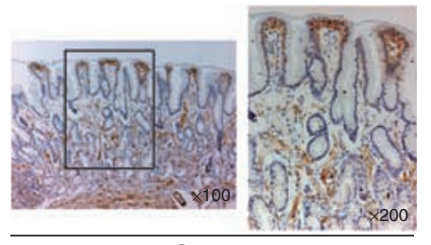

SMTN

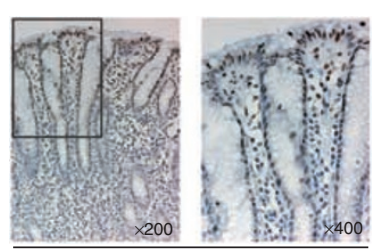

GLI2

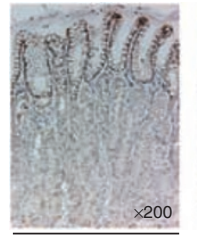

PDGFRB

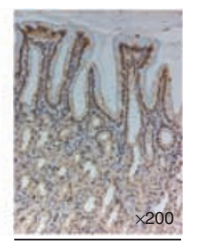

EDNRA

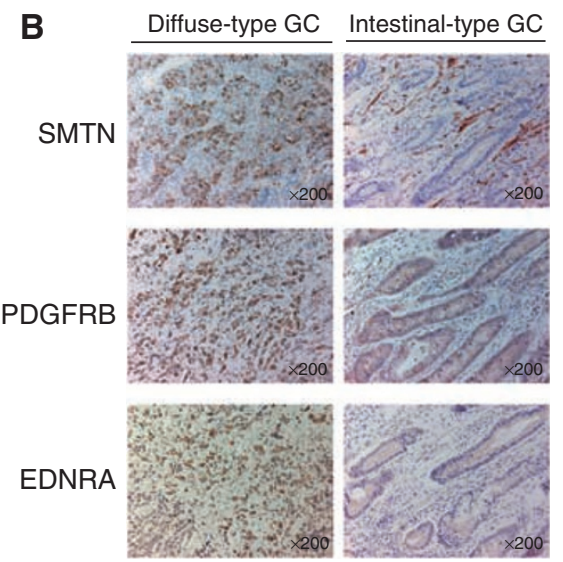

Figure 2 Immunohistochemistry of Hh downstream targets (GLII and GLI2) and mesenchymal-related genes (SMTN, PDGFRB, and EDNRA) in normal gastric mucosas and in gastric cancer tissues. (A) Both GLII and GLI2 are localised in the nuclei of the pit cells, and SMTN, PDGFRB, and EDNRA are stained preferentially in the cytoplasm or cell membrane of the pit cells. (B) SMTN, PDGFRB, and EDNRA are stained strongly in diffusetype gastric cancer cells compared with intestinal-type gastric cancer cells.

\section{Matrigel invasion assay}

Invasion of the GC HSC60 cells in vitro was measured by BD BioCoat $^{\mathrm{TM}}$ Matrigel $^{\mathrm{TM}}$ Invasion Chamber (6-well) (Becton Dickinson Biosciences, Bedford, MA, USA), according to the manufacturer's protocol. After SIP1 siRNA transfection, the cells were trypsinised and $2 \mathrm{ml}$ of cell suspension $\left(2 \times 10^{5}\right.$ cells ml $\left.^{-1}\right)$ was added in triplicate wells. For a cell growth assay, we counted the number of cells at $24 \mathrm{~h}$ after SIP1 siRNA transfection. For an invasion assay, the cells that passed through the filter into the lower wells were fixed and stained with $100 \%$ methanol and $1 \%$ Toluidine blue, respectively. The number of invading cells was counted through a microscope at $24 \mathrm{~h}$ after SIP1 siRNA transfection.

\section{RESULTS}

\section{Genome-wide mRNA expression profiling of primary intestinal-type and diffuse-type GCs}

To identify the specific genes for each type of GC, we selected 18 intestinal-type GCs and 12 diffuse-type GCs showing typical characteristics on the form of cell growth (clustered or scattered) and the degree of differentiation (well/moderate or poor), and performed microarray analysis for obtaining genome-wide mRNA expression profiles. First, we conducted unsupervised clustering analyses using some gene sets, which were differentially expressed depending samples. Most of the 12 diffuse-type GCs were able to separate from the 18 intestinal-type GCs (data not shown), suggesting the presence of a distinct difference on expression profiles between the two types. Next, to compile a gene list for identifying diffuse-type GC-specific signal pathways, we selected genes by comparing the expression levels of the two types. A gene was selected by Wilcoxon $U$-test $(P<0.05)$ from genes with more than a two-fold change on average. By this procedure, a total of 892 genes (704 genes specific to diffuse-type and 188 genes specific to intestinal-type) were identified. The result of a two-dimensional hierarchical clustering analysis of the 892 selected genes is shown in Supplementary Figure 1. In the 188 intestinal-type-specific genes, $C C N D E, E R B B 2$, and $G R B 7$, which have been reported to be amplified and overexpressed in intestinal-type GC (Yasui et al, 2001), were included. In the 704 diffuse-type-specific genes, three GLI1-target genes FOXM1, ISL1, and FOXF2 were included in accordance with our previous report (Fukaya et al, 2006). More interestingly, $C D H 1$ encoding an epithelial cell marker E-cadherin, which has been reported to be downregulated by an EMT regulator, TWIST, in breast cancer (Yang et al, 2004), was found only in intestinal-type GC, suggesting that diffuse-type GC may show EMT. In accordance with this suggestion, nine other mesenchymal-related genes (three muscle-related genes, SMTN, $L M O D$, and $M E F 2 C$; one blood-related gene, PDGFRB; one neuronal endocrine-related gene, EDNRA; two endotheliumrelated genes, SLIT2 and ROBO1; a target gene of an EMT regulator SIP1, WNT5A and its receptor, ROR2), were also found in the diffuse-type-specific gene list, although expression of EMT regulator genes (TWIST1, TWIST2, and SNAI2) other than SIP1 was never detected in the microarray platform used.

\section{Normal gastric pit cells and diffuse-type GCs possess mesenchymal-like gene expression}

In the gastric corpus, the epithelium consists of three tubular units from surface to base: a pit region containing mucus-secreting pit cells, an isthmus/neck region containing stem cells, and a gland region containing chief and parietal cells (Karam and Leblond, 1995). Intestinal-type GC is thought to develop from IM by transdifferentiation, whereas diffuse-type GC is derived from stem cell or the pit cell precursor (Ming, 1998; Yuasa, 2003). Therefore, genes specifically expressed in the diffuse-type may be expressed preferentially in the pit region among the three regions of the gastric mucosa. Accordingly, we prepared RNA from each region by LMD for RT - PCR as described in Materials and Methods. We conducted semi-quantitative RT-PCR analysis of Hh signallingand EMT-related molecules in the three regions of the gastric mucosa, IM, and two types of GC tissues. For semi-quantitative RT - PCR, we performed 25-35 cycles of PCR, and showed data within linear range.

Shown in Figure 1 are the results of semi-quantitative RT-PCR on eight Hh signalling genes (SHH, IHH, DHH, BOC, PTCH, SMO, GLI1, and GLI2), four GLI1 targets (ISL1, BMP4, FOXM1, and FOXA2), four EMT regulator genes (SIP1, SNAI2, TWIST1, and TWIST2), an epithelial cell marker ( $C D H 1)$, three mesenchymal markers $(C D H 2, V I M$, and $F N 1, \mathrm{M}-1$ in Figure 1), and nine other mesenchymal-related genes (SMTN, LMOD, MEF2C, PDGFRB, EDNRA, SLIT2, ROBO1, WNT5A, and ROR2, M-2 and $\mathrm{M}-3$ in Figure 1), in the RNA samples from the three regions of gastric mucosa, four IMs, eight intestinal-type GCs and 10 diffuse-type GCs.

In our previous report (Fukaya et al, 2006), preferential expression of most of the Hh signalling molecules (SHH, IHH, DHH, PTCH, SMO, GLI1, GLI2, ISL1, BMP4, FOXM1, and FOXA2) in diffuse-type GCs compared with IMs and intestinal-type GCs is confirmed by quantitative real-time RT - PCR in the same sample sets used in this paper. A new positive mediator of Hh signalling 
A

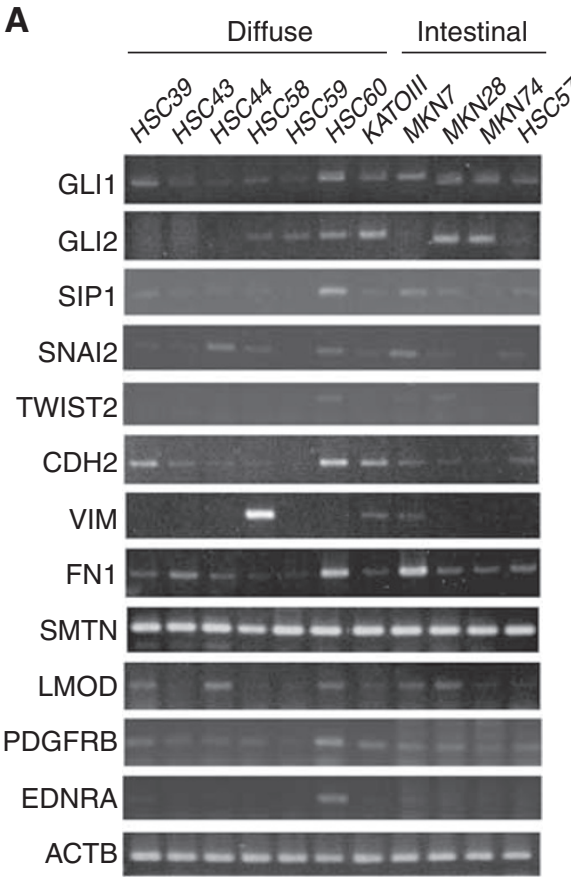

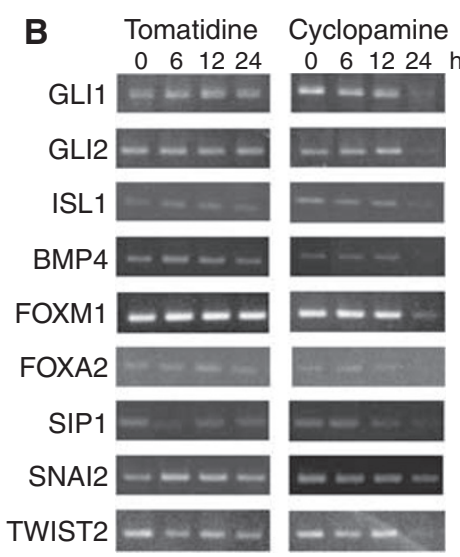

C

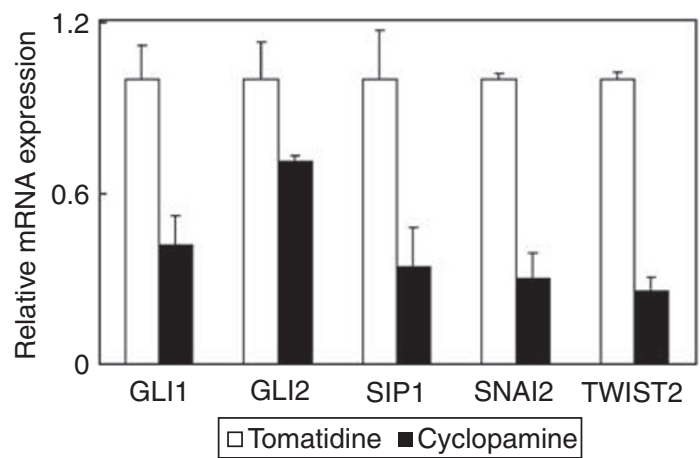

Figure 3 Effect of treatment of Hh-signal-specific inhibitor, cyclopamine on gastric cancer cells. (A) RT-PCR analyses of Hh- and EMT-related genes in I I gastric cancer cell lines. Only HSC60 cells most express Hh signalling, EMT regulator, and mesenchymal genes. (B) Cyclopamine treatment reduces mRNA of not only six Hh-downstream genes (GLII, GLI2, ISLI, BMP4, FOXMI, and FOXA2) but also three pit cell-expressing EMT regulators (SIPI, SNAI2, and TWIST2) and eight mesenchymal-related genes (CDH2, PDGFRB, EDNRA, ROBOI, WNT5A, ROR2, MEF2C, and SMTN). (C) Quantitative real-time RT-PCR analyses of Hh-downstream genes (GLII and GLI2) and EMT regulators (SIPI, SNAI2 and TWIST2) show the same results of the above semi-quantitative RTPCR. Results were calculated as mean + s.d. values from triplicate measurements of three separate experiments.

$B O C$ expressed highly in diffuse-type GCs, while another positive mediator $C D O$ expressed ubiquitously in the three regions of the gastric mucosa, IM, and two types of GC tissues (data not shown). The epithelial cell marker $C D H 1$ was downregulated in the diffusetype compared with the intestinal-type.

In normal tissue, among the four EMT regulators, SIP1, SNAI2, and TWIST2 were preferentially expressed in the pit region, whereas TWIST1 was expressed only in the gland region. In correspondence with SIP1, SNAI2, and TWIST2 expressions in the pit region, FN1, SMTN, LMOD, MEF2C, PDGFRB, EDNRA, SLIT2, ROBO1, WNT5A, and ROR2, preferentially expressed in the pit region, whereas $\mathrm{CDH} 2$ expressed in the gland region concordant with TWIST1 expression. In abnormal and malignant tissue, EMT regulators and mesenchymal-related genes were downregulated frequently in both the IMs and intestinaltype GCs, while those expressions were maintained in the diffuse-type GCs.

The results of RT-PCR analysis were confirmed by immunohistochemical analyses with GLI1, GLI2, SMTN, PDGFRB, and EDNRA antibodies. Both GLI1 and GLI2 were localised in the nuclei of the pit cells, and SMTN, PDGFRB, and EDNRA were stained preferentially in the cytoplasm or membranes of the pit cells (Figure 2A). These three genes were detected clearly in diffuse-type GC cells, but scarcely detected in intestinal-type GC cells (Figure 2B). These results suggest that, despite a differentiated epithelial cell, the gastric pit cell exhibits the mesenchymal phenotype, and that diffuse-type GC also maintains it. This suggests the presence of cross talk between the Hh and EMT signal pathways in both the pit cell and diffuse-type GC.

\section{Cross talk between Hh and EMT pathways in gastric pit} cells and in diffuse-type GCs

As shown in Figure 1, in GC tissues, expression patterns of Hhand EMT-related genes including a Hh-primary transcriptional target GLI1 and an EMT regulator SIP1 (indicated by a box) were most likely to be similar among the samples examined (only the no. 10 case in 10 diffuse-type GCs shows weak expression of these genes, whereas nos. 1, 2, 3, 5, and 6 cases in eight intestinaltype GCs showed weak). This observation suggested the presence of cross talk between Hh and EMT pathways.

To examine whether the EMT regulator gene is a downstream target of GLIs in Hh signalling, we first selected the GC cell line, which most-closely mimics the normal pit cell and diffuse-type GC phenotype in mRNA expression of Hh- and EMT-related genes among 11 GC-derived cell lines. As shown in Figure 3A, the diffuse-type GC cell lines except a HSC60 cell line (HSC39, HSC 43, HSC44, HSC58, HSC59, and KATOIII) appeared to have downregulated expression of a number of the Hh- and EMT-related genes. A tumour tissue provides various microenvironments (low nutrition, hypoxia, and so on) for tumour cell growth. In a tumour tissue, diffuse-type GC cells show scattered type growth by interaction with myofibroblasts. In a cell culture, three cell lines (HSC39, HSC58, and KATOIII) grow as spheroids. However, this characteristic could not explain the downregulation in the cell lines. Accordingly, the microenvironment and/or the interaction may be required for the activation of the Hh and EMT pathways. As shown in Supplementary Figure 2, both GLI1 and SIP1 were never upregulated in a culture with a low serum level. Recently, 
A

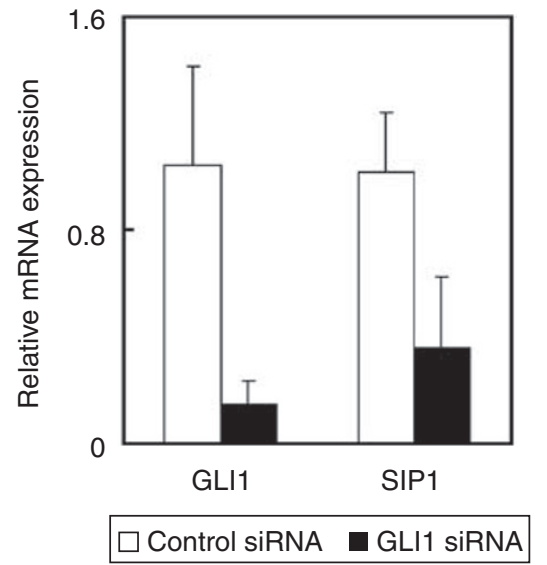

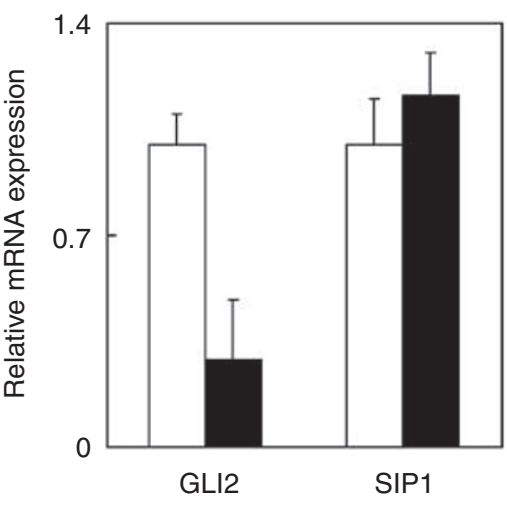

$\square$ Control siRNA $\mathbf{a L I} 2$ siRNA

B
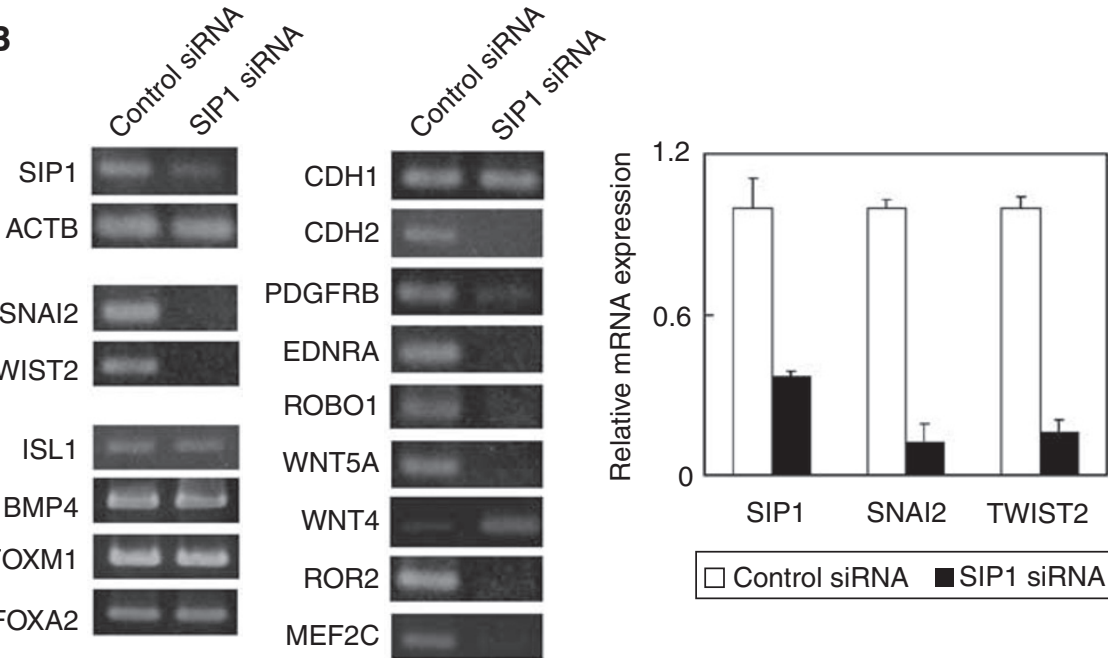

Figure 4 The EMT regulator gene SIPI is a mediator for mesenchymal-like gene expression through Hh signalling in diffuse-type gastric cancer cells. (A) Quantitative real-time RT-PCR analyses of SIPI mRNA in GLII or GLI2 siRNA-treated HSC60 cells. GLII siRNA reduces SIPI mRNA, whereas GLI2 siRNA does not reduce SIPI mRNA. Results were calculated as mean + s.d. values from triplicate measurements of three separate experiments. (B) Conventional and quantitative real-time RT-PCR analyses of EMT regulators, Hh target genes, and mesenchymal-related genes in SIPI siRNA-transfected HSC60 cells. In accordance with a decrease of SIPI mRNA, other EMT regulators (SNAI2 and TWIST2), and various mesenchymal-related genes (CDH2, PDGFRB, EDNRA, ROBO I, WNT5A, ROR2, and MEF2C) are downregulated. On the other hand, mRNA levels of Hh target genes (ISLI, BMP4, FOXM I, and FOXA2) and CDHI, an epithelial cell marker, are never affected, and WNT4, which is the other epithelial cell marker and involved in mesenchymal-epithelial transition (MET) (Stark et al, 1994), is clearly upregulated by SIPI siRNA treatment. Results of quantitative real-time RT-PCR were calculated as mean + s.d. values from triplicate measurements of three separate experiments.

hypoxia-inducing factor-1 (HIF-1) has been reported to activate or stabilise EMT regulators including TWIST and SNAIL (Gort et al, 2008; Sahlgren et al, 2008; Yang et al, 2008), but no report for such activation in GLIs has been found. Therefore, to date, the difference of EMT-related gene expression between cultured cells and primary tumours can be postulated partly by hypoxia. Although it was still unknown why the HSC60 cell line maintains the predisposition of both the pit cell and diffuse-type GC in expression of Hh signal- and EMT-related genes (Figure 3A), to verify the above-mentioned hypothesis, we examined the effects of treatment of an Hh signal pathway-specific inhibitor, cyclopamine (Cooper et al, 1998; Chen et al, 2002) in this cell line. The mRNA level of not only six Hh-downstream genes (GLI1, GLI2, ISL1, BMP4, FOXM1, and FOXA2) but also three pit cell-expressing EMT regulators (SIP1, SNAI2, and TWIST2) and their candidate downstream genes (CDH2, PDGFRB, EDNRA, ROBO1, WNT5A, ROR2, MEF2C, and $S M T N$ ) significantly decreased at $24 \mathrm{~h}$ after cyclopamine treatment compared with tomatidine, an inactive but structurally related compound (Figure $3 \mathrm{~B}$ ). The results of semiquantitative RT - PCR of five key transcription factors (GLI1, GLI2,
SIP1, SNAI2, and TWIST2) were confirmed by performing quantitative real-time PCR (Figure 3C).

Consecutively, GLI1 and GLI2 siRNA transfection were performed to investigate whether SIP1 is a downstream target of GLI. GLI1 siRNA transfection reduced the mRNA level of SIP1, whereas GLI2 siRNA transfection never affected it (Figure 4A). These results suggest that SIP1 is a downstream gene of GLI1 but not of GLI2, although a decrease of SIP1 protein could not be confirmed due to the low amount of SIP1 in HSC60 and the low specificity of anti-SIP1antibodies obtained commercially and by immunisation of our prepared-peptide sequences (data not shown). Next, we conducted SIP1 siRNA transfection to examine the downstream pathway of SIP1 (Figure 4B). SIP1 siRNA treatment reduced mRNAs of SIP1, two other EMT regulators (SNAI2, and TWIST2) and seven other genes (CDH2, PDGFRB, EDNRA, ROBO1, WNT5A, ROR2, and MEF2C). Although an epithelial cell marker $C D H 1$ was never affected by SIP1 siRNA treatment, WNT4, which has been reported to be involved in epithelial transition (Stark et al, 1994), was clearly upregulated by the treatment (Figure 4B, left panel). 
A

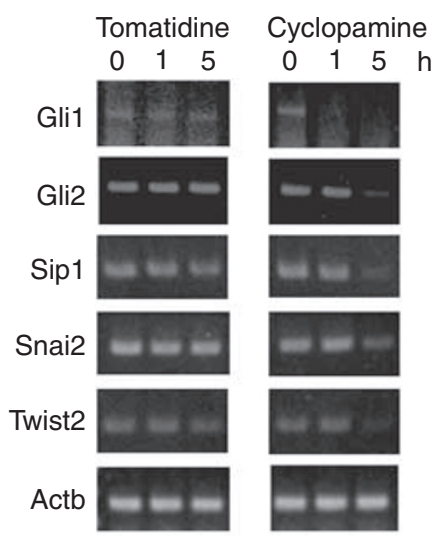

B

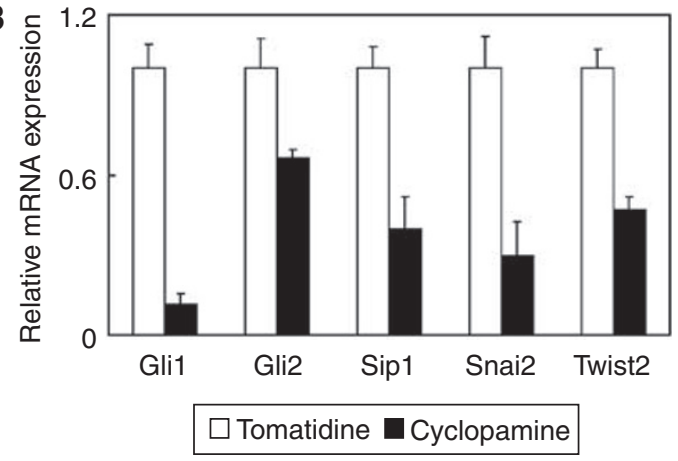

C

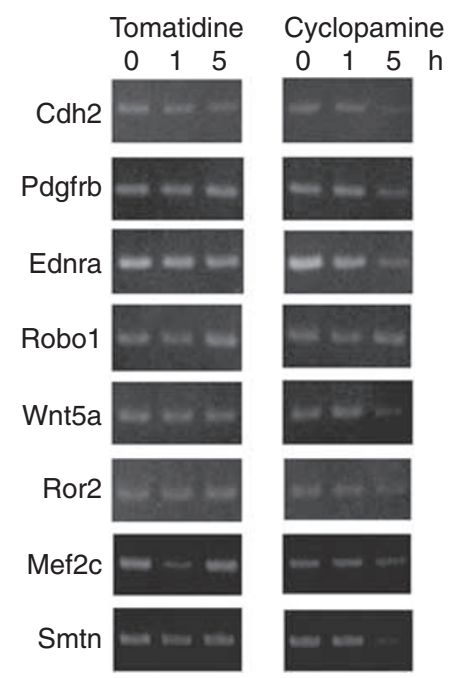

Figure 5 Cyclopamine treatment in mouse primary culture of the gastric epithelial cells. (A) In mice as well as in humans, not only Hh-downstream targets (Glil and Gli2) but also EMT regulators (Sip I, Snai2, and Twist2) are downregulated by cyclopamine treatment. (B) Quantitative real-time RTPCR analyses show the same results of the above conventional RT-PCR. Results were calculated as mean + s.d. values from triplicate measurements of three separate experiments. (C) Other mesenchymal-related genes (Cdh2, Pdgfrb, Ednra, Robol, Wnt5a, Ror2, Mef2c, and Smtn) are also downregulated by cyclopamine treatment. As a control, Actb is shown in Figure 5A.

As expected, the Hh target genes (ISL1, BMP4, FOXM1, and FOXA2) were never affected by SIP1 siRNA treatment (Figure 4B, left panel). The results of conventional RT-PCR in SIP1, SNAI2, and TWIST2 were verified by quantitative real-time PCR (Figure $4 \mathrm{~B}$, right panel). Those results of cyclopamine- (Figures 3B and 3C), GLI1 siRNA- (Figure 4A), and SIP1 siRNA (Figure 4B)- treatment suggested that some mesenchymal phenotypes in diffuse-type GC was exhibited by an EMT regulator SIP1 through Hh signalling.

The expression pattern of three ligands, Ihh (pit), Shh (gland), and Dhh (gland) in mouse gastric mucosa is similar to that in human beings, and Hh signal activation is found in both pit cells and parietal cells in murine glandular stomach (Van den Brink et al, 2001; Fukaya et al, 2006). To this end, to investigate the function of the Hh signal pathway in normal gastric mucosa, we examined the effects of cyclopamine treatment in mouse primary culture of gastric epithelial cells. Cyclopamine treatment reduced mRNAs of not only pit cell expressing-Hh signalling molecules (Gli1 and Gli2) but also EMT regulators (Sip1, Snai2, and Twist2) (Figure 5A). Quantitative real-time PCR analyses showed the same results (Figure 5B). Furthermore, mesenchymal-related genes (Cdh2, Pdgfrb, Ednra, Robo1, Wnt5a, Ror2, Mef2c, and Smtn) were also downregulated by cyclopamine treatment (Figure 5C). These data suggest that mesenchymal-like gene expression of the pit cell in both mice and humans is induced by $\mathrm{Hh}$ signal activation.

\section{Identification of cancer-specific Hh-downstream target genes}

An Hh-signal-specific inhibitor, cyclopamine, induces growth inhibition in Hh-activating GC cells; meanwhile in normal gastric epithelium, cyclopamine causes a block of pit cell differentiation followed by epithelial hyperplasia (Fukaya et al, 2006). For minimising the side effects in Hh-signal targeted cancer therapy, identification of cancer-specific Hh-downstream genes such as oncogenes is needed. Recently, evidence showing oncogene activation by cancer-linked DNA hypomethylation has accumulated (Feinberg et al, 2006). We previously reported 159 genes, including oncogenes, which could be activated by GC-linked DNA hypomethylation (Nishigaki et al, 2005). Among the gene list, RT-PCR of four oncogenes (RHO6, RHOB, ELK1, and MSX2) in surgical specimens was performed. All four genes were suppressed in normal gastric epithelium. Two genes, ELK1 and MSX2, of the four genes were found to express preferentially in diffuse-type GC (Figure 6A). Immunohistochemical analyses confirmed preferential expression of ELK1 and MSX2 proteins in diffuse-type GC cells compared with intestinal-type GC cells. Representative results are shown in Supplementary Figure 3. As shown in Figure 6B, cyclopamine treatment reduced mRNA of ELK1 and MSX2, suggesting both of the genes are regulated by $\mathrm{Hh}$ signalling. GLI1 siRNA treatment reduced mRNA of ELK1 and MSX2 (Figure 6B). These results suggest that ELK1 and MSX2 may be demethylated in diffuse-type GCs and then activated by GLI1. Next, we investigated whether knockdown of ELK1 and MSX2 using siRNA affects cell growth. Treatment of each siRNA of ELK1 and MSX2 induced growth inhibition (53 and $41 \%$, respectively) of HSC60 at 4 days after transfection (Figure 6C). Double transfection of these two siRNAs strongly inhibited cell growth (Supplementary Figure 4). These data suggest that blocking of both the ELK1 and MSX2 function could be an effective Hh-targeted cancer therapy.

\section{SIP1 is involved in GC cell invasion}

Finally, to investigate the biological implications of the Hh-EMT pathway in diffuse-type GC, Matrigel invasion assays were performed. Although no cell growth inhibition was observed at $24 \mathrm{~h}$ after SIP1 siRNA transfection, the number of invaded HSC60 cells was significantly decreased after SIP1 siRNA transfection compared with a control siRNA (Figure 7), suggesting that SIP1 was able to regulate genes critical to the invasion of diffuse-type GC cells. 
A

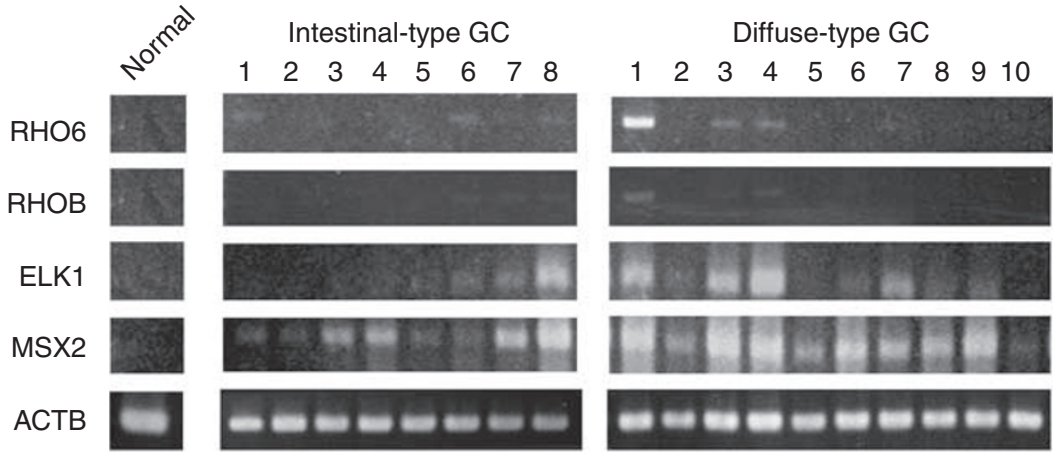

B
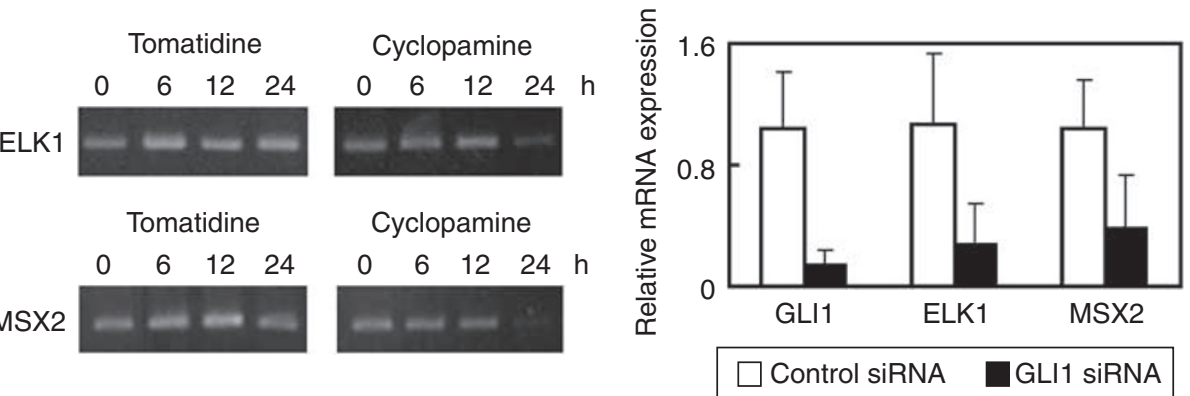

C
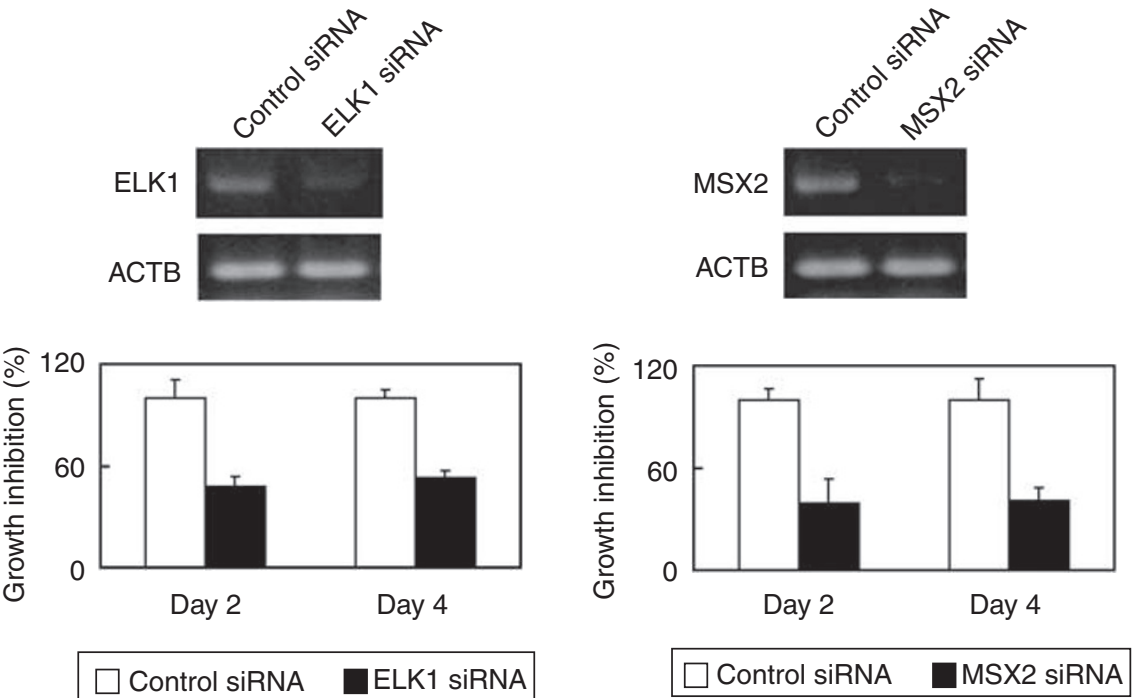

Figure 6 Identification of cancer-specific Hh-target genes. (A) RT-PCR analyses of gastric cancer-linked DNA hypomethylated candidate genes (RHO6, RHOB, ELKI, and MSX2) in normal gastric mucosa and cancer tissues. All four genes are suppressed in normal epithelium, and two genes ELKI and MSX2 of the four genes expressed preferentially in diffuse-type gastric cancer. (B) Cyclopamine (left panel) and GLII siRNA (right panel) treatment reduces ELKI and MSX2 mRNAs at $24 \mathrm{~h}$ after treatment. Results of real-time RT-PCR were calculated as mean + s.d. values from triplicate measurements of three separate experiments. As a control, Actb is shown in Figure 3B. (C) Treatment of ELKI siRNA and MSX2 siRNA induces growth inhibition of HSC60 cells (lower panel) in accordance with a decrease of ELKI and MSX2 mRNAs (upper panel). Results of cell growth inhibition assays were calculated as mean + s.d. values from triplicate measurements of three separate experiments. GC, gastric cancer.

\section{DISCUSSION}

Our present results suggest the presence of cross talk between $\mathrm{Hh}$ and EMT in gastric pit cells and in diffuse-type GC (Figure 8). The reason why gastric pit cells exhibit the mesenchymal phenotype, despite differentiating or differentiated epithelial cells, is unknown. Recently, we reported that GSDM/GSDMA was a target of RUNX3 and LMO1 in transforming growth factor-beta (TGF- $\beta$ ) signalling for apoptosis of gastric pit cells (Saeki et al, 2007). Transforming growth factor-beta is well known for inducing EMT as well as apoptosis of epithelial cells (Zavadil and Böttinger, 2005). Therefore, EMT may have a role in migration of the differentiating pit cells toward the gastric lumen.
To address the molecular mechanism of the cross talk between Hh and EMT, chromatin immunoprecipitation (ChIP)on-chip analysis is a hopeful tool for showing an in vivo direct interaction of the SIP promoter with GLI1, or of SIP1-downstream genes with SIP1. Although a GLI1 binding consensus sequence can be found about $1 \mathrm{~kb}$ upstream on the SIP1 promoter, there is no report showing a GLI1 antibody suitable for ChIP. As shown in Figure 6B, mRNAs of two GC-linked DNA hypomethylated oncogene candidates, ELK1 and MSX2, were clearly reduced by cyclopamine treatment and by GLI1 siRNA transfection. These results suggest that GLI1 or other GLI1-target transcriptional factors, including ISL1, FOXM1, FOXA2, and SIP1, may regulate 


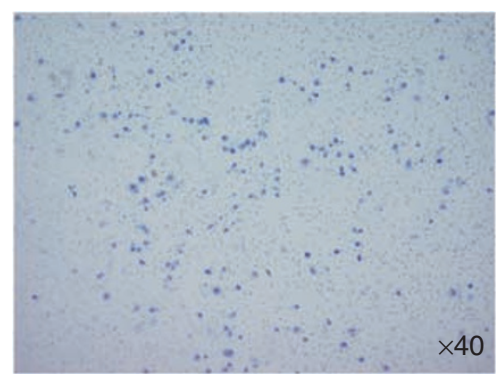

Control siRNA

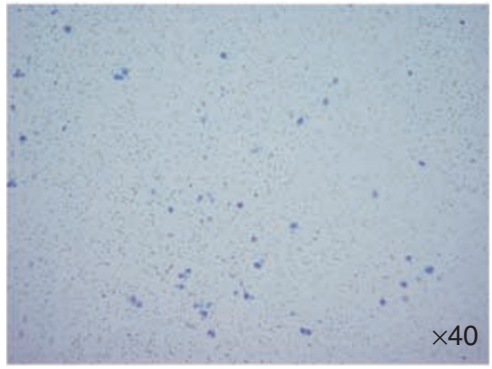

SIP1 siRNA

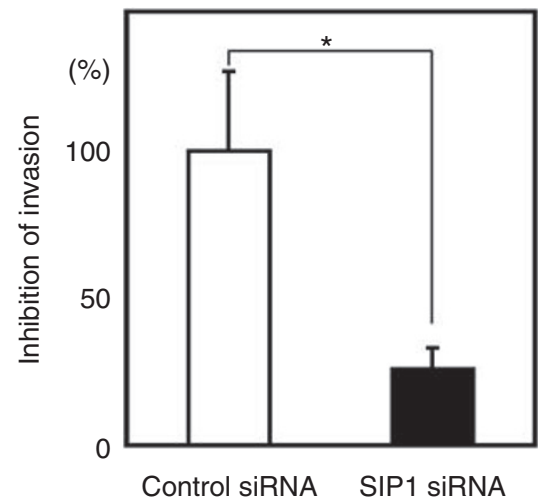

Figure 7 The EMT regulator gene SIPI involves gastric cancer cell invasion. Matrigel invasion assays indicate that SIPI siRNA transfection inhibits migration of HSC60 cells. Results were calculated as mean + s.d. values from triplicate measurements of three separate experiments. ${ }^{*} P<0.05$.

both ELK1 and MSX2. These issues remain, however, for future studies.

More than $70 \%$ of advanced GC patients show poor prognosis (Wohrer et al, 2004; Lordick and Siewert, 2005). Therefore, a new strategy or drug development is eagerly awaited. Advanced diffuse-type GC is known to frequently show peritoneal metastasis within 3 years. Our previous study (Fukaya et al, 2006) and this study show that the Hh signal is activated in most diffuse-type GCs, and that the Hh signal-specific inhibitor, cyclopamine, effectively suppressed invasion as well as growth of $\mathrm{Hh}$ signalactivated GC cells (Figure 7 and Fukaya et al, 2006). Therefore, diffuse-type GC could be a therapeutic target of Hh-pathwayspecific inhibitors, especially for the protection of peritoneal recurrence. Our proposed extensive Hh signal pathway containing an EMT pathway could contribute to new drug development. As shown in Figure 8, two transcription factors, GLI1 and SIP1, seem to be the key molecules for the Hh-EMT pathway in diffuse-type GC, because GLI1 regulates cell growth-related genes, such as ELK1, MSX2, and FOXM1 (Kim et al, 2006; Yoshida et al, 2007), and because SIP1 regulates cell invasion-related genes such as EMT regulators (TWIST2 and SNAI2) (Taki et al, 2003; Yang et al,

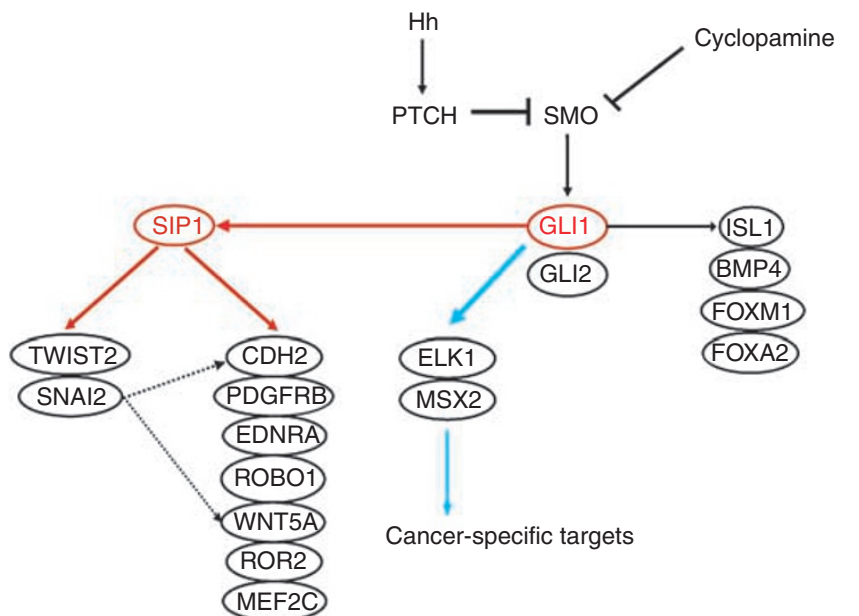

Figure 8 Hypothetical schema of cross talk between Hh and EMT signal pathways in both pit cells and diffuse-type gastric cancers. A transcriptional cross talk between $\mathrm{Hh}$ and EMT is indicated by red arrows, and that between $\mathrm{Hh}$ and cancer-specific genes (ELKI and MSX2) is indicated by blue arrows. Broken arrows that are headed from SNAI2 to $\mathrm{CDH} 2$ and WNT5A are based on a previous report (Taki et al, 2003).

2004), PDGFRB (Singh et al, 2007), and WNT5A (Kurayoshi et al, 2006). An increasing number of genes including oncogenes are found to be normally methylated at promoters but hypomethylated and activated in the corresponding tumours (Feinberg et al, 2006). These include R-RAS and MASP in GC (Akiyama et al, 2003; Nishigaki et al, 2005), MAGE1 in melanoma (De Smet et al, 1996), S100A4 in colon cancer (Nakamura and Takenaga, 1998), PAX2 in endometrial cancer (Wu et al, 2005), DNMT3A in testicular germ cell tumours (Ishii et al, 2007), and various genes in pancreatic cancer (Sato et al, 2003). ELK1 and MSX2, encoding transcription factors, may belong to this class of oncogene. Double transfection of ELK1 and MSX2 siRNAs to diffuse-type GC cells showed strong cell growth inhibition compared with a single transfection (Figure 6C and D, and Supplementary Figure 4), suggesting that ELK1 and MSX2 act independently on GC cell growth. Therefore, a genomewide search of the transcriptional downstream target gene of ELK1 and MSX2 in addition to GLI1 and SIP1 is thought to be important for future identification of molecular targets of diffuse-type GC.

In summary, our study showed that genome-wide mRNA expression profiling provides some hints for identifying specific signal pathways, their cross talk, and some molecules aberrantly expressed in the pathways in a certain type of cancer. However, the sensitivity varies among microarray platforms, and most or all major platforms have an insufficient sensitivity to detect a key molecule, such as a growth factor or transcription factor, which acts with a small expression. Improvement of the sensitivity is needed for future cancer transcriptome.

\section{ACKNOWLEDGEMENTS}

This study was supported in part by the programme for promotion of Fundamental Studies in Health Sciences of the National Institute of Biomedical Innovation, in part by a Grant-in-Aid for the Third Comprehensive 10-Year Strategy for Cancer Control and 20-12 from the Ministry of Health, Labour and Welfare of Japan, and in part by a Research Grant of the Princess Takamatsu Cancer Research Fund. $\mathrm{H}$ $\mathrm{O}, \mathrm{MF}$, and NI were awardees of Research Resident Fellowships from the Foundation for Promotion of Cancer Research.

Supplementary Information accompanies the paper on British Journal of Cancer website (http://www.nature.com/bjc) 


\section{REFERENCES}

Akiyama Y, Maesawa C, Ogasawara S, Terashima M, Masuda T (2003) Celltype-specific repression of the maspin gene is disrupted frequently by demethylation at the promoter region in gastric intestinal metaplasia and cancer. Am J Pathol 163: 1911 - 1919

Aoyagi K, Tatsuta T, Nishigaki M, Akimoto S, Tanabe C, Omoto Y, Hayashi S, Sakamoto H, Sakamoto M, Yoshida T, Terada M, Sasaki H (2003) A faithful method for PCR-mediated global mRNA amplification and its integration into microarray analysis on laser-captured cells. Biochem Biophys Res Commun 300: $915-920$

Chen JK, Taipale J, Cooper MK, Beachy PA (2002) Inhibition of Hedgehog signaling by direct binding of cyclopamine to Smoothened. Genes Dev 16: $2743-2748$

Cooper MK, Porter JA, Young KE, Beachy PA (1998) Teratogen-mediated inhibition of target tissue response to Shh signaling. Science 280: $1603-1607$

Crew KD, Neugut AI (2006) Epideiology of gastric cancer. World J Gastroenterol 12: 354-362

De Smet C, De Backer O, Faraoni I, Lurquin C, Brasseur F, Boon T (1996) The activation of human gene MAGE-1 in tumor cells is correlated with genome-wide demethylation. Proc Natl Acad Sci USA 93: 7149-7153

Feinberg AP, Ohlsson R, Henikoff S (2006) The epigenetic progenitor origin of human cancer. Nat Rev Genet 7: $21-33$

Fukaya M, Isohata N, Ohta H, Aoyagi K, Ochiya T, Saeki N, Yanagihara K, Nakanishi Y, Taniguchi H, Sakamoto H, Shimoda T, Nimura Y, Yoshida T, Sasaki H (2006) Hedgehog signal activation in gastric pit cell and in diffuse-type gastric cancer. Gastroenterology 131: 14-29

Gort EH, van Haaften G, Verlaan I, Groot AJ, Plasterk RHA, Shvarts A, Suijkerbuijk KPM, van der Wall E, Raman V, van Diest PJ, Tijsterman M, Vooijs M (2008) The TWIST oncogene is a direct target of hypoxiainducible factor-2a. Oncogene 27: $1501-1510$

Hohenberger P, Gretschel S (2003) Gastric cancer. Lancet 362: 305 - 315

Ishii T, Kohu K, Yamada S, Ishidoya S, Kanto S, Fuji H, Moriya T, Satake M, Arai Y (2007) Up-regulation of DNA-methyltransferase $3 \mathrm{~A}$ expression is associated with hypomethylation of intron 25 in human testicular germ cell tumors. Tohoku J Exp Med 212: $177-190$

Karam S, Leblond CP (1995) Origin and migratory pathways of the eleven epithelial cell types present in the body of the mouse stomach. Microsc Res Tech 31: $93-214$

Kim IM, Ackerson T, Ramakrishna S, Tretiakova M, Wang IC, Kalin TV, Major ML, Gusarova Ga, Yoder HM, Costa RH, Kaliniechenko VV (2006) The Forkhead Box mi transcription factor stimulates the proliferation of tumor cells during development of lung cancer. Cancer Res 66: 2153-2161

Kobayashi K, Nishioka M, Kohno T, Nakamoto M, Maeshima A, Aoyagi K, Sasaki H, Takenoshita S, Sugimura H, Yokota J (2004) Identification of genes whose expression is upregulated in lung adenocarcinoma cells in comparison with type II alveolar cells and bronchiolar epithelial cells in vivo. Oncogene 23: 3089-3096

Kountouras J, Zavos C, Chatzopoulos D (2005) New concepts of molecular biology on gastric carcinogenesis. Hepatogastroenterology 52: 1305-1312

Krishnan V, Elberg G, Tsai MJ, Tsai SY (1997) Identification of a novel sonic hedgehog response element in the chicken ovalbumin upstream promotertranscription factor II promoter. Mol Endocrinol 11: 1458-1466

Kurayoshi M, Oue N, Yamamoto H, Kishida M, Inoue A, Asahara T, Yasui W, Kikuchi A (2006) Expression of Wnt-5a is correlated with aggressiveness of gastric cancer by stimulating cell migration and invasion. Cancer Res 66: 10439-10448

Lauren P (1965) The two histological main types of gastric carcinoma: diffuse and so-called intestinal-type carcinoma. An attempt at a histoclinical classification. Acta Pathologica Et Microbiologica Scandinavica 64: $31-49$

Lordick F, Siewert JR (2005) Recent advances in multimodal treatment for gastric cancer: a Review. Gastric Cancer 8: 78-85

Maxwell Parkin D (2001) Global cancer statistics in the year 2000. Lancet Oncol 2: $533-543$

Ming SC (1998) Cellular and molecular pathology of gastric carcinoma and precursor lesions: a critical review. Gastric Cancer 1: $31-50$

Nakamura N, Kobayashi K, Nakamoto M, Kohno T, Sasaki H, Matsuno Y, Yokota J (2006) Identification of tumor markers and differentiation markers for molecular diagnosis of lung adenocarcinoma. Oncogene 25: $4245-4255$

Nakamura N, Takenaga K (1998) Hypomethylation of the metastasisassociated S100A4 gene correlates with gene activation in human colon adenocarcinoma cell lines. Clin Exp Metastasis 16: $471-479$
Nishigaki M, Aoyagi K, Danjoh I, Fukaya M, Yanagihara K, Sakamoto H, Yoshida T, Sasaki H (2005) Discovery of aberrant expression of R-RAS by cancer-linked DNA hypomethylation in gastric cancer using microarrays. Cancer Res 65: 2115-2124

Saeki N, Kim DH, Usui T, Aoyagi K, Tatsuta T, Aoki K, Yanagihara K, Tamura M, Mizushima H, Sakamoto H, Ogawa K, Ohki MK, Shiroishi T, Yoshida T, Sasaki H (2007) GASDERMIN, suppressed frequently in gastric cancer, is a target of LMO1 in TGF- $\beta$-dependent apoptotic signalling. Oncogene 26: 6488-6498

Sahlgren C, Gustafsson MV, Jin S, Poellinger L, Lendahl U (2008) Notch signaling mediates hypoxia-induced tumor cell migration and invasion. Proc Natl Acad Sci USA 105: 6392-6397

Sato N, Maitra A, Fukushima N, van Heek NT, Matsubayashi H, IacobuzioDonahue CA, Rosty C, Goggins M (2003) Frequent hypomethylation of multiple genes overexpressed in pancreatic ductal adenocarcinoma. Cancer Res 63: 4158-4166

Schier S, Wright NA (2005) Stem cell relationships and the origin of gastrointestinal cancer. Oncology 69: 9-13

Singh PK, Wen Y, Swanson BJ, Shanmugam K, Kazlauskas A, Cerny RL, Gendler SJ, Hollingsworth MA (2007) Platelet-derived growth factor receptor beta-mediated phosphorylation of MUC1 enhances invasiveness in pancreatic adenocarcinoma cells. Cancer Res 67: 5201-5211

Stark K, Vainio S, Vassileva G, McMahon AP (1994) Epithelial transformation of metanephric mesenchyme in the developing kidney regulated by Wnt-4. Nature 372: 679-683

Taki M, Kamata N, Yokoyama K, Fujimoto R, Tsutsumi S, Nagayama M (2003) Downregulation of Wnt4 and upregulation of Wnt5a expression by epithelialmesenchymal transition in human squamous carcinoma cells. Cancer Sci 94: 593-597

Tatematsu M, Ichinose M, Miki K, Hasegawa R, Kato T, Ito N (1990) Gastric and intestinal phenotypic expression of human stomach cancers as revealed by pepsinogen immunohistochemistry and mucin histochemistry. Acta Pathologica Japonica 40: 494-504

Teh MT, Wong ST, Neill GW, Ghali LR, Philpott MP, Quinn AG (2002) FOXM1 is a downstream target of Gli1 in basal cell carcinomas. Cancer Res 62: 4773 - 4780

Tenzen T, Allen BL, Cole F, Kang JS, Krauss RS, McMahon AP (2006) The cell surface membrane protein Cdo and Boc are components and targets of the Hedgehog signaling pathway and feedback network in mice. Dev Cell 10: $647-656$

Van den Brink GR, Hardwick JC, Tytgat GN, Brink MA, Ten Kate FJ, Van Deventer SJ, Peppelenbosch MP (2001) Sonic hedgehog regulates gastric gland morphogenesis in man and mouse. Gastroenterology 121: $317-328$

Wohrer SS, Raderer M, Hejna M (2004) Palliative chemotherapy for advanced gastric cancer. Ann Oncol 15: 1585-1595

Wu H, Chen Y, Liang J, Shi B, Wu G, Zhang Y, Wang D, Li R, Yi X, Zhang H, Sun L, Shang Y (2005) Hypomethylation-linked activation of PAX2 mediates tamoxifen-stimulated endometrial carcinogenesis. Nature 438: $981-987$

Yang J, Mani SA, Donaher JL, Ramaswamy S, Itzykson RA, Come C, Savangner P, Gitelman I, Richardson A, Weinberg RA (2004) Twist, a master regulator of morphogenesis, plays an essential role in tumor metastasis. Cell 117: 927 -939

Yang MH, Wu MZ, Chiou SH, Chen PM, Chang SY, Liu CJ, Teng SC, Wu KJ (2008) Direct regulation of TWIST by HIF-1a promotes metastasis. Nat Cell Biol 10(3): 295-305. doi: 10.1038/ncb1691

Yao S, Lum L, Beachy P (2006) The Ihog cell-surface proteins bind hedgehog and mediate pathway activation. Cell 125: $343-357$

Yasui W, Oue N, Kuniyasu H, Ito R, Tahara E, Yokozaki H (2001) Molecular diagnosis of gastric cancer: present and future. Gastric Cancer 4: $113-121$

Yoshida Y, Wang IC, Yoder HM, Davidson NO, Costa RH (2007) The forkhead box M1 transcription factor contributes to the development and growth of mouse colorectal cancer. Gastroenterology 132: 1420-1431

Yuasa Y (2003) Control of gut differentiation and intestinal-type gastric carcinogenesis. Nat Rev Cancer 3: 592-600

Zavadil J, Böttinger EP (2005) TGF-beta and epithelial-to-mesenchymal transitions. Oncogene 24: 5764-5774

Zhang W, Kang JS, Cole F, Yi MJ, Krauss RS (2006) Cdo functions at multiple points in the Sonic Hedgehog pathway, and Cdo-deficient mice accurately model human holoprosencephaly. Dev Cell 10: 657-665 